\title{
Digital Application Specific Integrated Circuit Design
}

\section{Llovizna Miranda, Valparaiso University - SIST Intern}

\section{Triple Modular Redundancy Tool for System Verilog}

\section{Background: TMR}

Single radiation effects can be transient and static. The charge collected from radiation has a prompt (drift) and a slow (diffusion) component. This current spike causes disparate results: SEU, SET, SEB, MBU, and more. A system level SEE mitigation technique, used to overcome, is the triple module redundancy (TMR)

TMR for system Verilog (TMR4SV), written in Python, is the first step towards design IP triplication. It allows various triplication methods for flip flops, clocks, and logic.

\section{Technology}

PyCharm, Linux, HSpice

\section{Objectives and characteristics}

1. User interface to choose methodology.

2. Constraint file is modified if clocks or logic are triplicated.

3. Clock and reset pins are re-named to user's preference.

4. All components are triplicated as chosen by the user. The designated folder should have a new file named module TMR.sv.

5. Input file's name is modified to have modules name: module.sv.

Overall, the use of the triplication alleviates the radiation's effect and prevents upsets within a state's transition.

Acknowledgments: Thank you to Farah Fahim, Troy England, Leandro Stefanazzi, Manuel Blanco Valentin, SIST Committee, and LSMRCE Committee
Visuals of various Triplication Methods
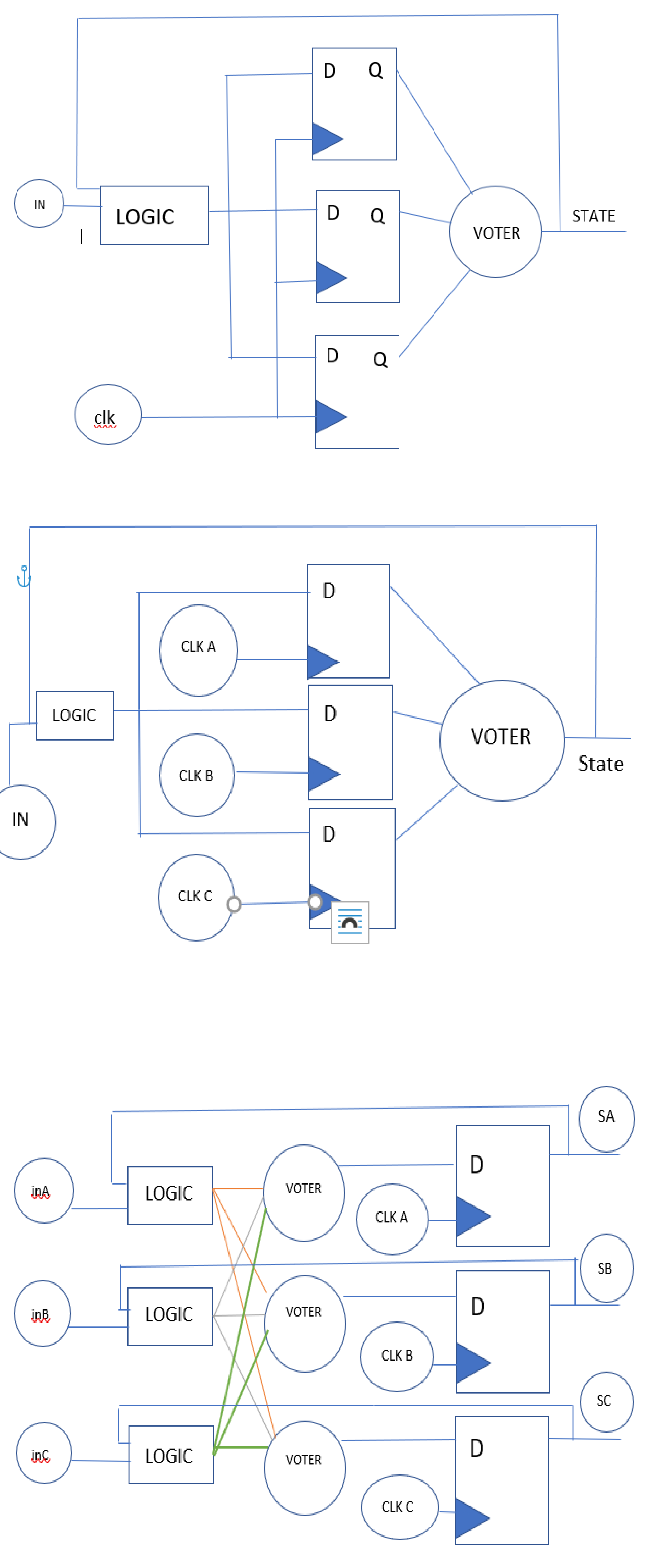

\section{VHDL for Pile Up Control}

Flowchart for Pile Up Control
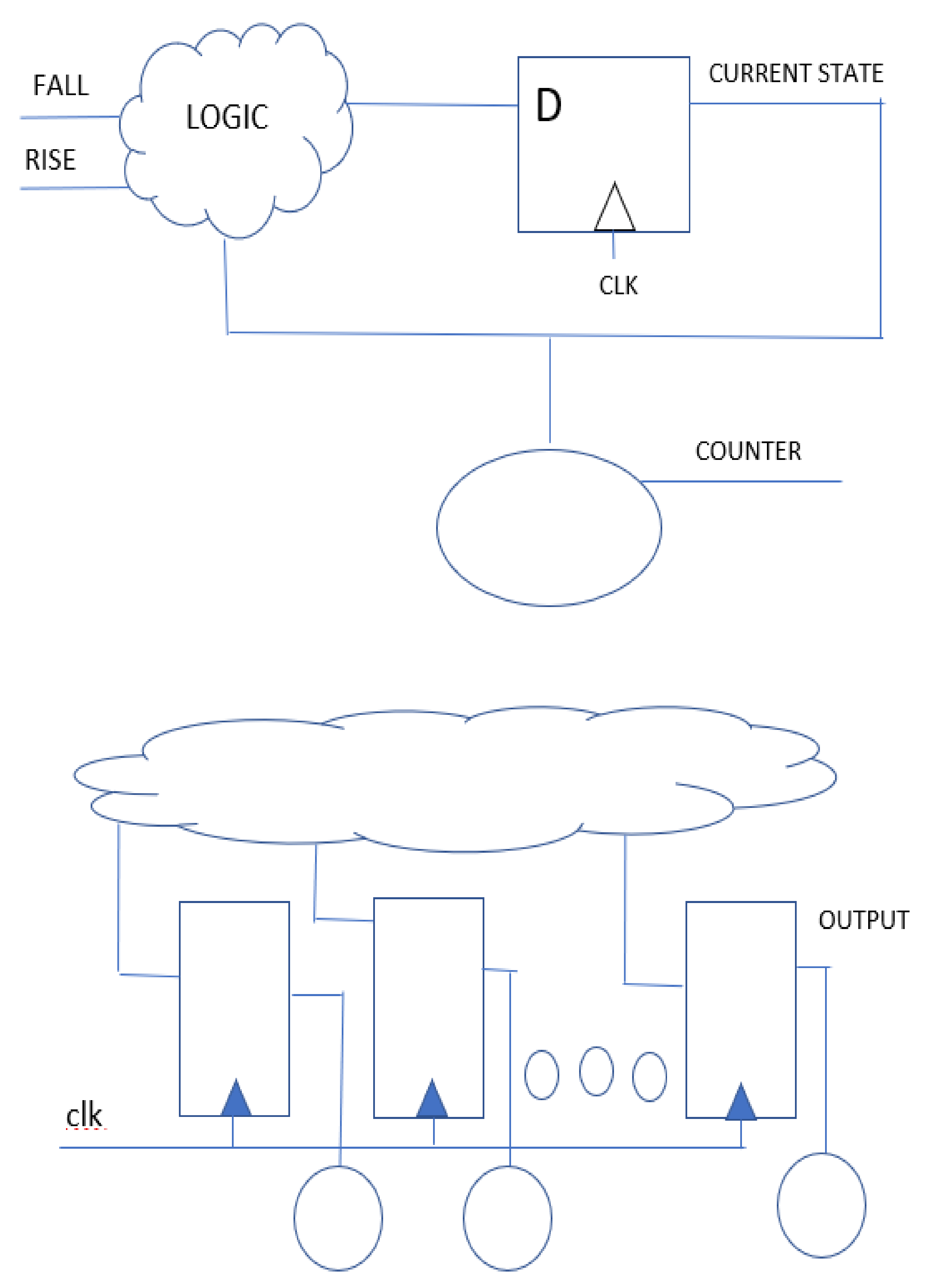

Technology ModelSim, Linux
Background for Pile Up Control

The asic analog memory can hold up to 16 values of signals per channel, and the pile up control logic allows to serialize read and write from it.

The pile up control written in VHSIC Hardware Description Language (VHDL) Code enables the use of 16 capacitors. This is done by reading 3 input signals: integrate, readint, rst.

The input integrate generates two pulses. For every two pulses, a capacitor (C) goes high for $\mathrm{C}<\mathrm{i}+1>$.

- Logic: Identify integrate's rise and fall.

- FSM: Determines state based on logic.

- The FSM's output, current state, is then included into the logic.

- Counter: Counts up until the signal readin goes high.

- Flip Flops: They represent the capacitors $\mathrm{C}<\mathrm{i}>$.

The VHDL Code includes an Entity, Architecture, 3 Processes, and a Case Statement.

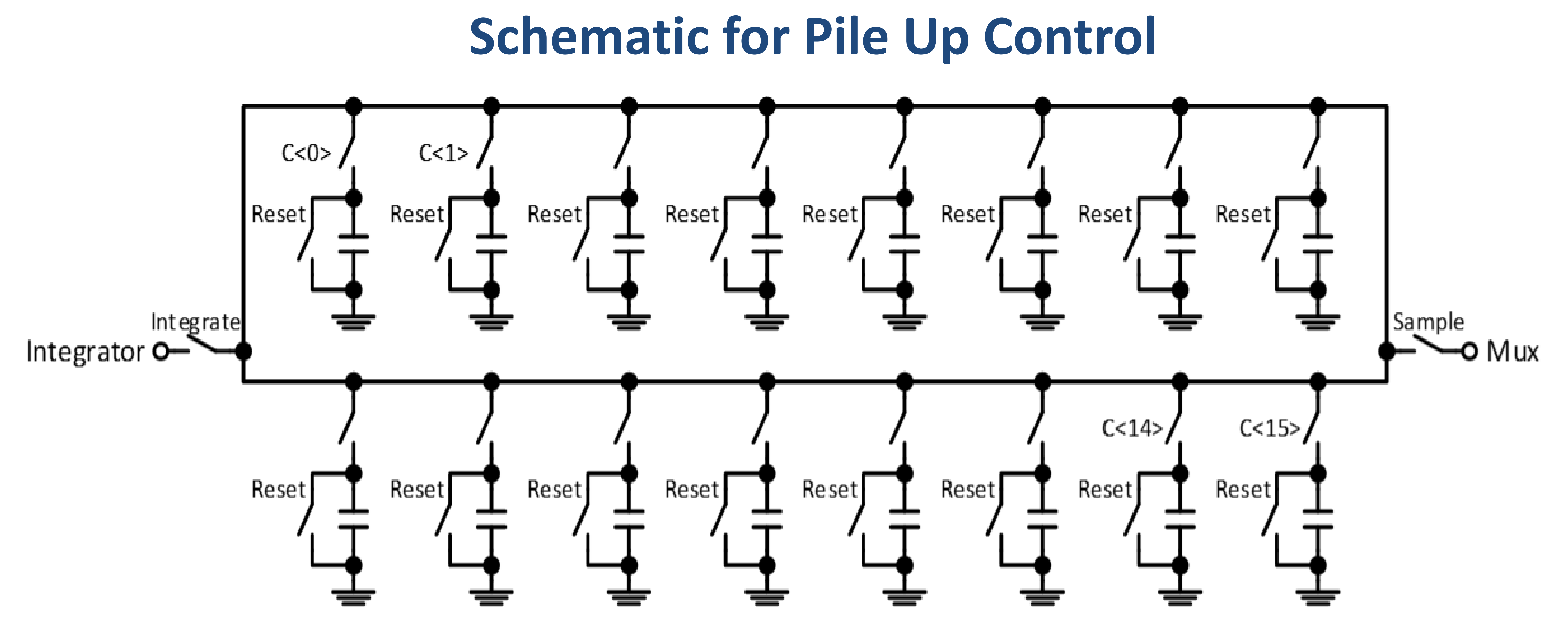




\section{Triple Module Redundancy and Pile Up Control}

Llovizna Miranda, SIST Intern, Farah Fahim, Supervisor-

Subtitles - Helvetica / Arial Bold 50pt RGB 0,76,151

Background:

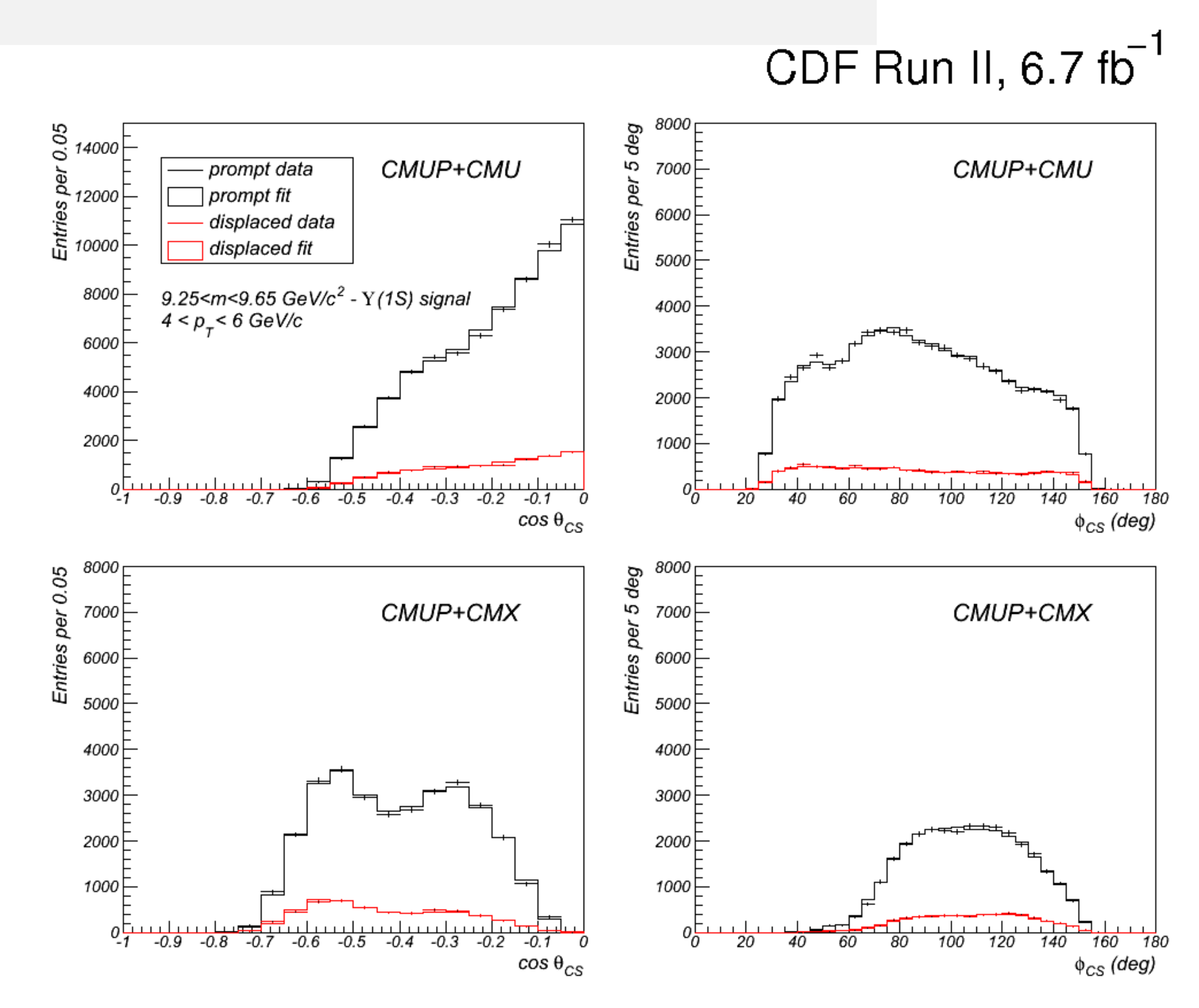

